\title{
Wind Tunnel Test of an RPV with Shape-Change Control Effector and Sensor Arrays
}

\author{
David L. Raney and Randolph H. Cabell ${ }^{\ddagger}$ \\ NASA Langley Research Center \\ Hampton, VA 23681 \\ William G. Barnwell ${ }^{\dagger}$ and S. Todd Lion ${ }^{\S}$ \\ North Carolina State University \\ Raleigh, NC 27695
}

\author{
Adam R. Sloan ${ }^{\dagger}$ \\ Stanford University \\ Stanford, CA 94305 \\ Bret A. Hautamaki ${ }^{\S}$ \\ University of Michigan \\ Ann Arbor, MI 48104
}

\begin{abstract}
A variety of novel control effector concepts have recently emerged that may enable new approaches to flight control. In particular, the potential exists to shift the composition of the typical aircraft control effector suite from a small number of high authority, specialized devices (rudder, aileron, elevator, flaps), toward larger numbers of smaller, less specialized, distributed device arrays. The concept envisions effector and sensor networks composed of relatively small high-bandwidth devices able to simultaneously perform a variety of control functions using feedback from disparate data sources. To investigate this concept, a remotely piloted flight vehicle has been equipped with an array of 24 trailing edge shape-change effectors and associated pressure measurements. The vehicle, called the Multifunctional Effector and Sensor Array (MESA) testbed, was recently tested in NASA Langley's 12-ft Low Speed wind tunnel to characterize its stability properties, control authorities, and distributed pressure sensitivities for use in a dynamic simulation prior to flight testing. Another objective was to implement and evaluate a scheme for actively controlling the spanwise pressure distribution using the shape-change array. This report describes the MESA testbed, design of the pressure distribution controller, and results of the wind tunnel test.
\end{abstract}

\section{INTRODUCTION}

Recent discoveries in material science and fluidics have been used to create a variety of novel effector devices that may enable new approaches to aerospace vehicle flight control. Potential exists to shift the composition of future aircraft control effector suites from a relatively small number of high authority, specialized devices (rudder, aileron, elevator, flaps), toward increasingly larger numbers of smaller, less specialized, distributed effector and sensor device arrays. ${ }^{1}$ Such effector arrays might operate in a decentralized fashion on local data from a distributed pressure sensor or accelerometer network to perform highly distributed tasks such as load alleviation, flutter suppression, separation control, or pressure regulation, while simultaneously performing more centralized stability augmentation and maneuver control tasks using feedback from a conventional inertial measurement unit and air data sensor suite. Future aerospace vehicles might use large networks of sensor and effector devices in this capacity, thereby augmenting or replacing conventional control surfaces.
The presumed benefits of such a shift toward distributed effector systems are speculative and come at some cost, particularly in the form of increased system complexity. In addition to the aforementioned distributed control objectives, potential benefits include reduced fuel consumption, enhanced maneuverability, reconfigurability, health monitoring, failure tolerance and mission adaptability. Whether distributed systems will be able to buy their way onto future flight vehicles is likely to depend upon a great number of mission-specific factors and trade offs. But their development and demonstration at the fundamental research level will expand the design space and present options that may enable new missions and new capabilities, or enhance existing functions.

Although several research efforts at universities, government labs and industry are underway to develop and characterize novel effector devices including synthetic jets, shape-change blisters, and micro flaps, relatively few activities address the incorporation of large groups of such devices into aerospace vehicle flight control systems. This research seeks to develop flight

\footnotetext{
${ }^{\square}$ Research scientist, Dynamics and Control Branch, Senior Member AIAA.

${ }^{\ddagger}$ Research scientist, Structural Acoustics Branch, Senior Member AIAA.

Graduate Student, Mechanical and Aerospace Engineering Department, Member AIAA.

$\S$ Undergraduate, Mechanical and Aerospace Engineering Department, Member AIAA.
} 
control concepts for aerospace vehicles that incorporate measurements from distributed sensor arrays and issue commands to large numbers of distributed effectors to simultaneously perform multiple control functions. Control algorithms are developed and evaluated using a computer simulation model and wind tunnel testing of a small remotely piloted aircraft.

\section{BACKGROUND}

Prior work by Park and Green focused on optimizing the placement of distributed shape-change "bump effector" devices over the surface an advanced aircraft configuration for maneuver control purposes by applying automatic differentiation software to a potential flow analysis model. ${ }^{2}$ They applied these bump devices to Lockheed Martin's Innovative Control Effector (ICE) configuration in a conceptual design. A follow-on investigation by Padula and Rogers examined the use of genetic algorithms for determining placement of the shape-change devices. ${ }^{3}$ These efforts yielded a suite of surface bump control effectors for the ICE configuration. A related investigation by Raney and Montgomery developed a flight dynamic simulation and control design for the vehicle using this bump effector suite. ${ }^{4}$ The study found that the effector suite offered promise for seamless aircraft flight control in a low-rate maneuver mode that might be useful for stealth purposes, but that the authority of the surface bump devices was not sufficient to replace the conventional effector suite for this vehicle.

An unrelated investigation by Bieniawaski, Kroo, and Lee developed a testbed that used distributed microtrailing edge effectors (MiTEs) together with distributed accelerometers in a flutter suppression control system. ${ }^{5,6}$ Wind tunnel tests demonstrated successful flutter suppression using a novel control design that was generated by a "reinforcement learning" policy search technique. The investigation demonstrated the potential benefits of control through the use of a large number of small, mechanically simple, distributed effector devices, and developed a unique approach to control synthesis that was specifically tailored for distributed effector and sensor arrays.

A series of investigations that were funded under the DARPA/AFRL/NASA/Northrop Grumman Smart Wing Program have demonstrated the potential of distributed actuation systems to create gap-less, hinge-less continuous mold line control surfaces for adaptive wing designs. ${ }^{7,8}$ In particular, an investigation by Wang, et al addressed the design of distributed actuation systems to generate continuous shape-change deflections of the trailing edge of an airfoil. ${ }^{9}$ A number of material and actuation system concepts were examined, including shape-memory alloys (SMAs), piezo stacks, and ultrasonic motors. The pros and cons of each system were examined and a final design was generated that used ten distributed high-bandwidth ultrasonic motors to warp a seamless airfoil trailing edge into a variety of continuous geometries with the potential to produce maneuver control moments. In this case, the distributed shape-change effector system provides the airfoil with greater adaptability and functionality than could be achieved by a single large aileron effector occupying the same area.

The present investigation focuses on the use of a small remotely piloted vehicle as a testbed for the development of flight control algorithms using distributed effector and sensor arrays. The testbed is equipped with a total of 24 trailing edge shape-change effector devices and associated pressure measurements. The flight vehicle, called the Multifunctional Effector and Sensor Array (MESA) testbed, was built by NC State University with support from NASA's Aircraft Morphing Program. The vehicle has been tested in Langley's 12-ft Low Speed Tunnel to characterize its basic stability properties, control authorities, and distributed pressure responses to effector deflections for use in a dynamic simulation model prior to flight testing. An additional objective of the wind tunnel test was to implement and evaluate a scheme for regulating the spanwise pressure distribution on the testbed using the distributed sensor and effector array. The ability of the system to achieve and regulate a range of commanded spanwise pressure distributions in the presence of turbulence, flight condition perturbations, and device failures was examined.

This report describes the MESA remotely piloted vehicle (RPV), the design of the spanwise pressure distribution controller for use with the model's trailing edge effector and sensor array, and the results of both phases of the wind-tunnel test, consisting of open-loop characterization of the flight vehicle and closed-loop evaluation of the pressure distribution control design.

\section{EXPERIMENTAL RPV MODEL}

The MESA RPV was created by modifying a flight vehicle that had been originally designed, constructed, and flight-tested by a team of aerospace engineering seniors at NC State University during the 2001-2002 term. The original vehicle, named Thunderstruck, was similar in planform and appearance to the NASA-Boeing BWB design, although its two large vertical stabilizers were a notable departure. The configuration is shown in Figure 1, along with a view of the final prototype during its graduation flight. The vehicle used a symmetric NACA 0015 airfoil and had a wingspan of 9.4 feet, root chord of 4.9 feet, and takeoff weight of 39 pounds. The vehicle was designed to cruise at 80 miles per hour with a predicted stall speed of 30 miles per hour. An AMT Mercury Turbojet engine provided 14.82 pounds of available thrust. The aircraft was capable of carrying five pounds of payload. 

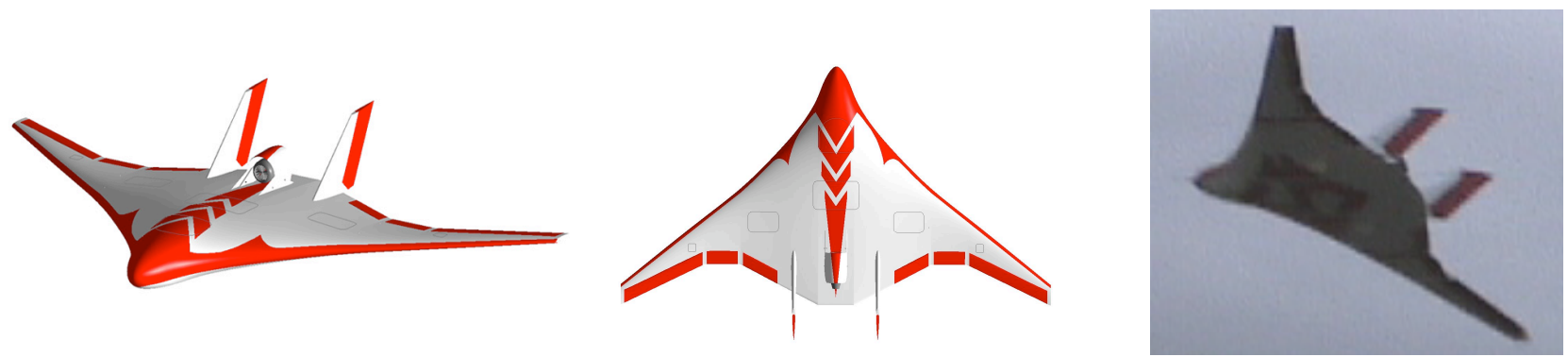

Figure 1. Isometric and planform views of Thunderstruck, with a photo of the vehicle during its graduation flight.

For the purposes of this investigation, Thunderstruck was transformed into the MESA RPV testbed under a cooperative agreement with NC State. New wings were designed and fabricated, each of which included a distributed array of 12 shape-change trailing edge effector devices and 12 distributed pressure measurements. The design of the shape-change devices composing the trailing edge array required several iterations.

\section{Shape-Change Effector Array}

Initially, the hope was to create a completely seamless shape-change trailing edge array such as the one designed in [9], but the weight and complexity of the resulting design was not suitable for this flight vehicle. Instead, it was decided to create a discretized approximation of a continuous mold line fully morphable trailing edge array by dividing the wing trailing edge into twelve small hingeless shape-change control surfaces able to bend in the vertical plane. Each device consists of two thin spring steel skin sections that are split at the trailing edge of the wing. The spring steel plates, having a thickness of 0.007 ", are warped by a lightweight hobby-class Hobbico CS-5 servomotor through a pullpull wire linkage; the opposing surface restores the actuated surface to its neutral position. Each effector segment measures 1.5 " wide $x$ " long, and is capable of deflecting through a range of \pm 15 degrees. This design allowed the array to be constructed of lightweight, inexpensive, commonly available and reliable supplies. Figure 2 shows a side view of the prototype effector design in the undeflected and fully deflected positions. ${ }^{10}$

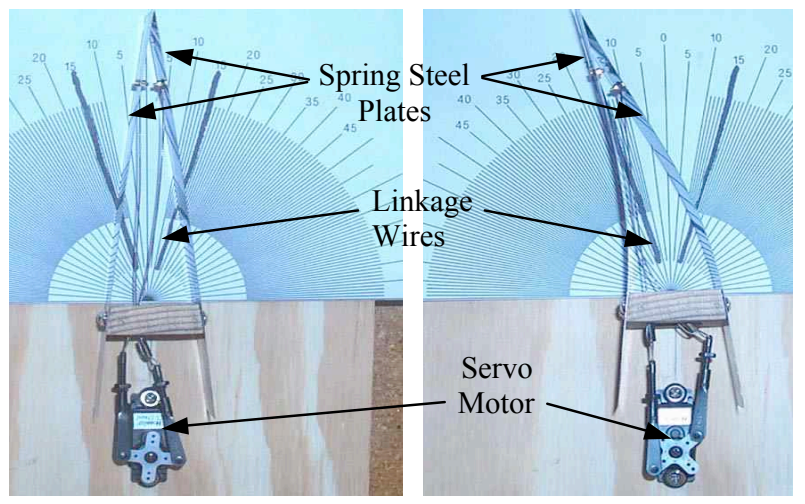

Figure 2. Side view of shape-change effector prototype.
The placement and number of effector devices that were incorporated into the MESA testbed was influenced by several factors. Due to accessibility issues within the existing aircraft fuselage, it was decided that the shapechange array should be confined to the removable wing sections. Wing thickness and chord limited the outboard placement of the devices. Refraining from extending the array into the outboard-most wing area also left space for a conventional aileron that could be used in conjunction with the remaining conventional surfaces to trim and control the testbed during takeoff, landing, and other non-research functions. Potential flow analysis generated linear aerodynamic force and moment estimates that were used in flight dynamic simulation studies to identify an installation region that would provide control authorities comparable to the conventional effector suite. These considerations lead to the final design decision to include twelve devices on each wing. The modified wing panels increased the RPV weight by $3 \mathrm{lb}$.

The MESA RPV is shown in Figure 3. In addition to the shape-change effector suite, the conventional aileron, elevon and rudder control effectors are apparent in the figure. Shown in Figure 4 is a panel model of the vehicle that was used in the potential flow analysis to generate preliminary estimates of stability and control characteristics for the flight dynamic simulation model. The potential flow results will be compared with wind tunnel measurements later in this report. Table 1 summarizes the flight vehicle characteristics. Further detail regarding design, fabrication, and preliminary testing of the shapechange effector array is provided in [10].

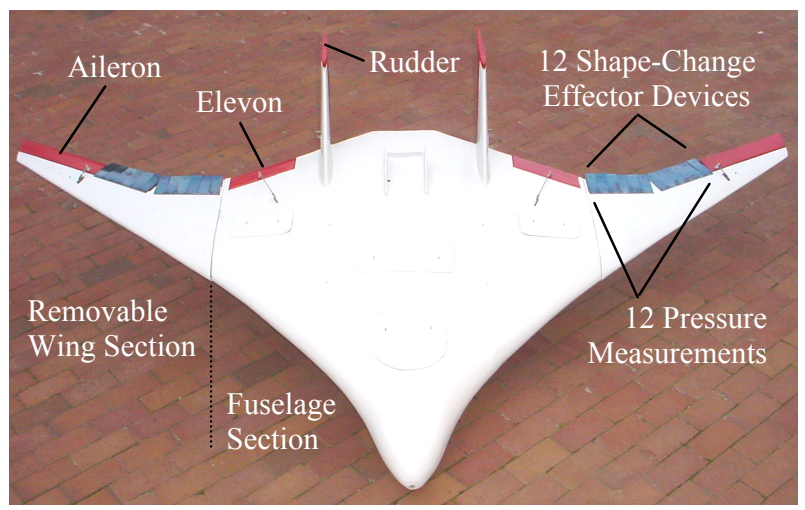

Figure 3. MESA RPV with modified wing panels. 


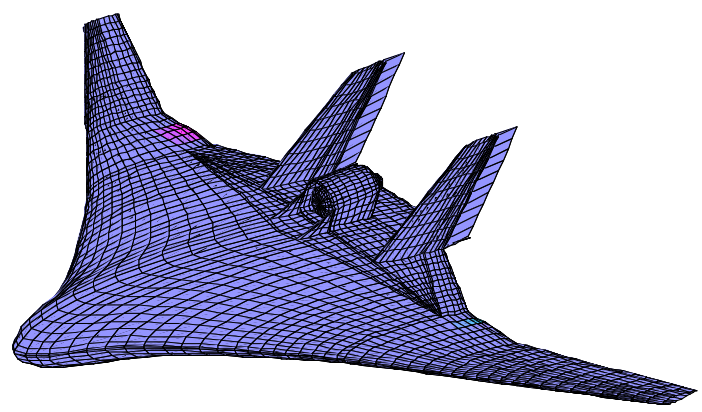

Figure 4. Potential flow panel model used to develop preliminary stability and control estimates.

\begin{tabular}{|l|c|}
\hline \multicolumn{1}{|c|}{ Parameter } & Value \\
\hline Takeoff Weight, Ib & 42 \\
Wing Area, $\mathrm{ft}^{2}$ & 17.77 \\
Span, $\mathrm{ft}$ & 9.38 \\
Mean Chord, $\mathrm{ft}$ & 2.86 \\
Vcruise, ft/s & 117 \\
Vstall, ft/s & 44 \\
Inertias, slug ft & \\
$\quad$ Ixx & 0.0808 \\
Iyy & 1.6362 \\
Izz & 2.3832 \\
Ixz & 0.0900 \\
\hline
\end{tabular}

Table 1. MESA flight vehicle characteristics

\section{Effector Deployment Modes and Shapes}

The effector array could be operated in either openloop or closed-loop command modes. The open-loop mode allowed commands to be generated using one of three settings. The first setting permitted independent deflections of individual effector segments. The second setting permitted the selection of one of six static trailing edge shapes, in which each effector took part in approximating a portion of the shape. A total of six shapes were implemented consisting of a constant offset of the trailing edge, a linearly sloped offset, half sine wave, whole sine wave, a 1.5-cycle sine wave, and finally an alternating plus/minus deflection. Photos of the left wing of the model with effector segments deployed in trailing edge shapes 1, 2, 3, and 6 are shown in Figure 5. The third open-loop setting permitted the array to be driven dynamically with an arbitrary deflection time history.

The closed-loop command mode drove the effector positions with a real-time hardware-in-the-loop feedback control system designed to achieve a commanded pressure distribution at the spanwise taps. The feedback control algorithm will be presented later in this report.

\section{Distributed Pressure Measurements}

Pressure measurement taps were placed upstream of each effector to investigate the potential to control the spanwise pressure distribution with the shape-change array. Locations of the taps were determined using a

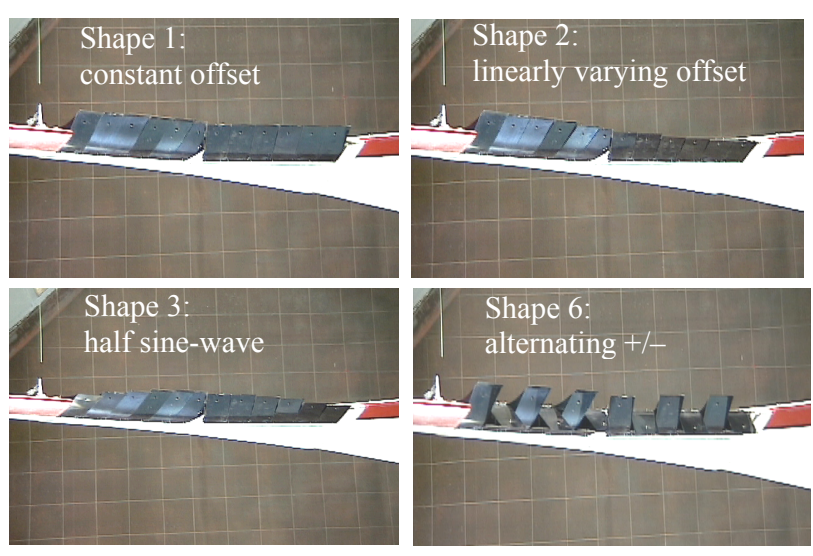

Figure 5. Effector array segments deployed in trailing edge shapes 1,2,3 and 6 .

potential flow prediction for the region of maximum pressure sensitivity to control surface deflection. Surface pressure taps were positioned 4.05" upstream of the trailing edge of each effector segment, for a total of 24 pressure taps, 12 per wing. The taps were equally spaced in the spanwise direction at intervals of 1.5 " with the first tap located 0.75 " from the root chord of the removable wing section shown in Figure 3. The taps measured 0.040 " in diameter and were connected to a Pressure Systems Inc. Electronic Scanning Pressure (ESP) module through 0.040 " diameter nylon tubing.

The ESP module was placed inside the model to minimize the pressure measurement lag associated with tubing length. The ESP had a range of $\pm 10 " \mathrm{H}_{2} \mathrm{O}$, and was capable of electronically multiplexing up to 32 independent pressure measurements. The ESP was located inside a thermostatically controlled heater box within the model to eliminate the influence of temperature fluctuations on the pressure transducers. Measurements from the 24 pressure taps on the model were electronically scanned and multiplexed by the ESP module at a time interval of $1.5 \mathrm{~ms}$, so the measurement at each tap was updated every $36 \mathrm{~ms}$.

\section{Control Interface}

The distributed pressure measurement and effector array control interface was designed and implemented using dSpace ${ }^{\mathrm{TM}}$ hardware-in-the-loop computer components and software together with Matlab's Simulink ${ }^{\mathrm{TM}}$ programming environment and Real-Time Workshop ${ }^{\mathrm{TM}}$ code generation package. The real time control process was implemented with a step size of $0.75 \mathrm{~ms}$.

The experimental setup included the ability to control all moveable surfaces of the model remotely using the dSpace real-time interface. The dSpace system included a dedicated Power PC $750 \mathrm{MHz}$ processor, 16-bit A-to-D and D-to-A boards, four digital I/O serial connections and a software interface designed to permit 
real-time hardware-in-the-loop execution of control algorithms. The dSpace system was located in the wind tunnel control room. The system not only provided the ability to control the model effectors with open-loop commands, but also to drive them with closed-loop signals from the pressure distribution controller. To achieve this capability it was necessary to implement the data acquisition software that drove the ESP module within the dSpace real-time control algorithm. Four BASIC- $X^{\mathrm{TM}}$ servo serial boards were used to translate position commands from the dSpace system into pulse train signals for the effector servos. Each board could drive up to eight servos. The boards were located within the model and were remotely commanded via the dSpace serial connections using RS232 protocol.

\section{FACILITY AND TEST CONDITIONS}

The model was tested in NASA Langley's 12-ft Low Speed Tunnel, operated by the Vehicle Dynamics Branch of the Airborne Systems Competency. The 12-Foot LowSpeed Tunnel is an atmospheric pressure, open circuit tunnel enclosed in a 60 -foot diameter sphere. The test section is octagonal, having a width and height of 12 feet and a length of 15 feet with each octagonal side measuring 5 feet. The maximum operating dynamic pressure is $\mathrm{q}=7 \mathrm{psf}(\mathrm{V}=77 \mathrm{ft} / \mathrm{sec}$ at standard sea level $)$, for a Reynolds number of approximately $490,000 \mathrm{ft}^{-1}$.

In this experiment, the model was tested over a range of angle of attack (AOA) from -2 to 20 degrees and sideslip from -6 to 6 degrees, and at dynamic pressure of $5 \mathrm{psf}$, corresponding to tunnel speed of $45 \mathrm{mph}$. The model's nominal flight speed is $80 \mathrm{mph}$, and this testing was conducted at approximately half that speed due to limitations associated with heating of the tunnel drive system. The model was mounted on a sting that exited through the lower surface of the fuselage. The mounting system included an internal 6-component strain gauge balance to provide force and moment data. Figure 6 shows the aircraft mounted in the test section.

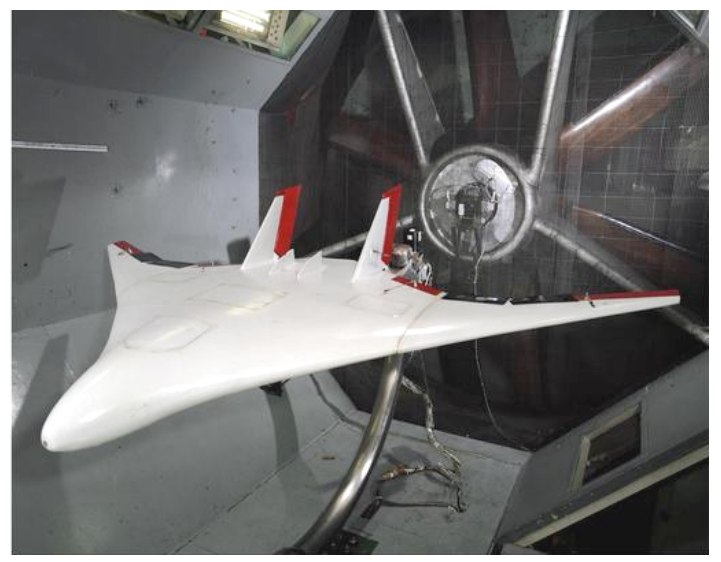

Figure 6. MESA RPV mounted in the NASA Langley 12-Foot Wind Tunnel.

\section{FORCE AND MOMENT RESULTS}

Force and moment data obtained from the test were compared with potential flow predictions that had been generated prior to the test. The potential flow analysis was performed using the PMARC code ${ }^{11}$ with the panel model shown in Figure 4. Potential flow predictions for coefficient of lift and pitching moment vs. angle of attack are compared with wind tunnel data in Figure 7. Moments are referenced to the aircraft center of gravity located 26 inches aft of the nose.

The gradual stall break shown in the upper plot of Figure 7 is typical of blended wing-body configurations. Linear aerodynamic predictions based on potential flow analysis assume inviscid, irrotational, unseparated flow, and so become increasingly unreliable as the vehicle approaches stall. The vehicle trims in $80 \mathrm{mph}$ cruise at approximately 4 degrees $\mathrm{AOA}$, but trims on approach and landing at approximately 9 to 10 degrees. The wind tunnel results indicate a serious reduction in static pitch stability at or near the approach condition.

Potential flow predictions for coefficients of yaw and roll vs. sideslip at the trim angle of attack of 4 degrees are shown in Figure 8. Both of these coefficients exhibit stable trends with sideslip angle. The potential flow analysis appears to provide reasonable predictions for the lateral/directional stability coefficients at the cruise condition, and these characteristics are very similar at the landing condition.

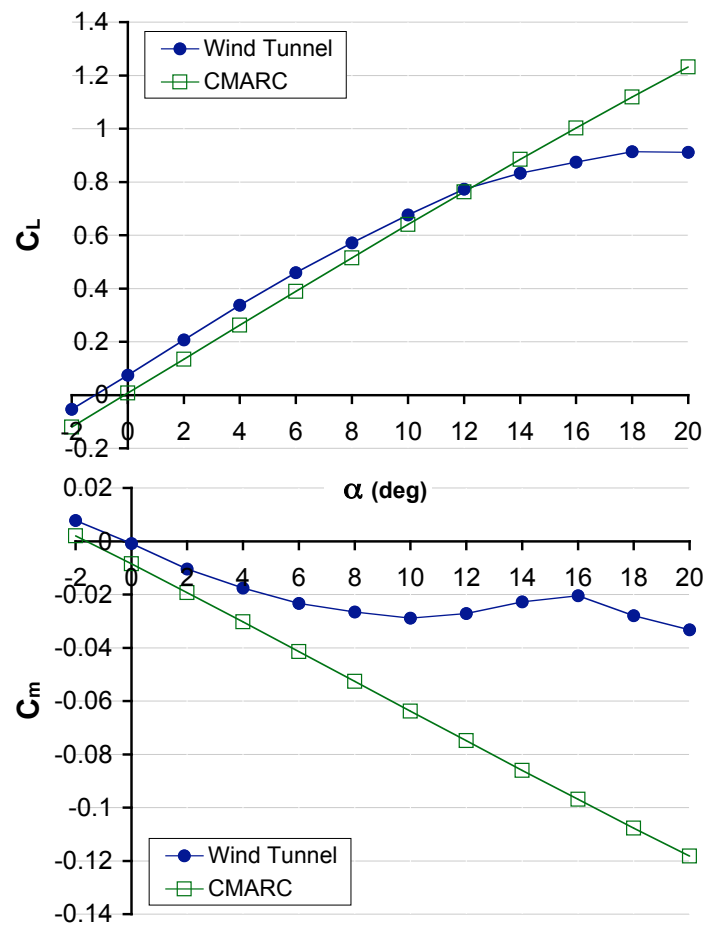

Figure 7. Comparison of wind tunnel data with PMARC predictions for lift (top) and pitching moment (bottom). 


\section{Control Authorities - Conventional Effectors}

The conventional effectors were tested over their full range of motion in 2-degree increments at AOAs of 0, 4, 8, and $12 \mathrm{deg}$. Deflection limits for the elevon, aileron, and rudder surfaces are $\pm 20, \pm 15$, and $\pm 14 \mathrm{deg}$, respectively. Positive deflections are defined as trailing edge down for symmetric elevons, right trailing edge up for ailerons, and trailing edge right for rudders.

Control moment vs. surface deflection angle are shown in Figure 9 for an AOA of $4 \mathrm{deg}$. Primary authorities for pitch due to symmetric elevon, roll due to aileron, and yaw due to rudder are shown at the top of the figure. Secondary (adverse and proverse) moment effects are shown in the plot at the bottom. The plots suggest that the conventional effector suite will provide adequate control authority. Pitch and roll moments due to rudder deflections are not negligible, and will be taken into account in the flight control system design. These data along with additional measurements that were collected during the wind tunnel test have been used in a dynamic simulation to verify that conventional effector suite will provide adequate control authority for typical flight maneuvers. The conventional effectors will be used during non-research portions of flight and as a backup to the shape-change effector array if needed.
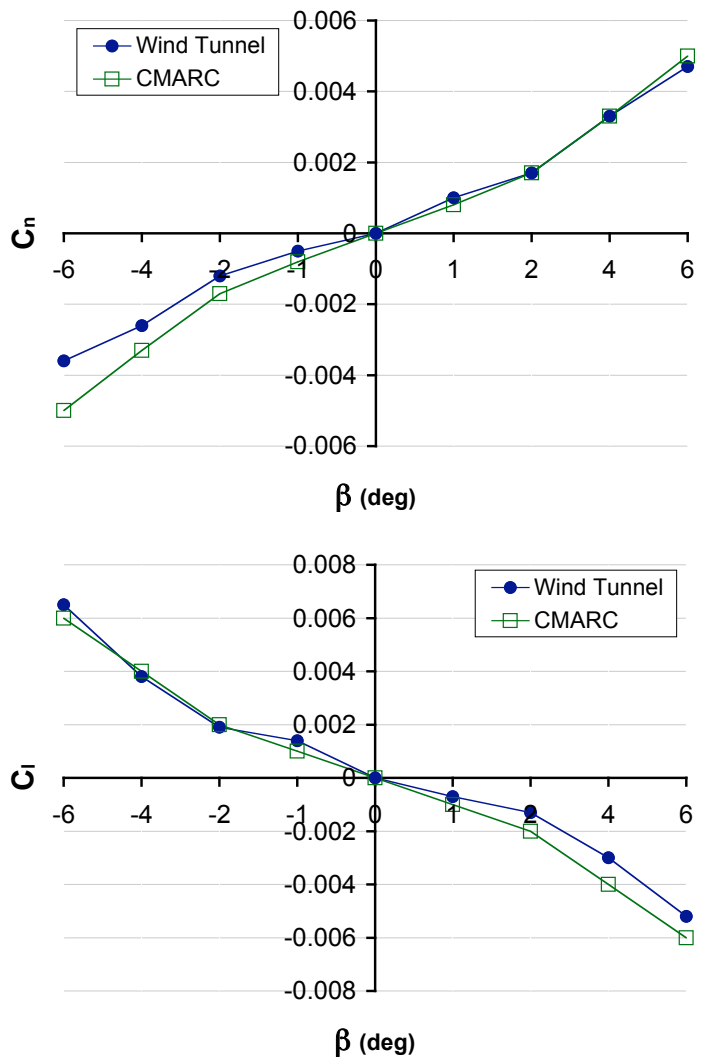

Figure 8. Comparison of wind tunnel data with PMARC predictions for yawing moment (top) and rolling moment (bottom) vs. sideslip, $\mathrm{AOA}=4 \mathrm{deg}$.

\section{Control Authorities - Individual Devices}

Control effector deflection sweeps were performed for each of the 24 devices that composed the trailing edge shape-change effector array. Angle of attack and control deflection increments were necessarily coarse to limit the size of the test matrix. Each device was tested over its full deflection range of -15 to +15 degrees in 5 degree increments at four angles of attack $(0,4,8$, and 12 degrees). Remote control of all effector settings through the dSpace console in the tunnel control room permitted automation of the test procedure, which greatly improved the ability to rapidly cover a large test matrix.

Figure 10 shows the pitch, roll, and yaw authority for effector number six, as numbered from inboard to outboard, on the right wing at an angle of attack of 4 degrees. The positive sense of deflection for all the effector array devices is defined as trailing edge up. The behavior is fairly linear and typical of that observed for all the trailing edge devices. Potential flow predictions of control moments produced by deflection of the individual devices were also developed, and these were over-predicted by roughly a factor of 2 .

Linear fits to the wind tunnel moment data were used to generate control authority derivatives for each of

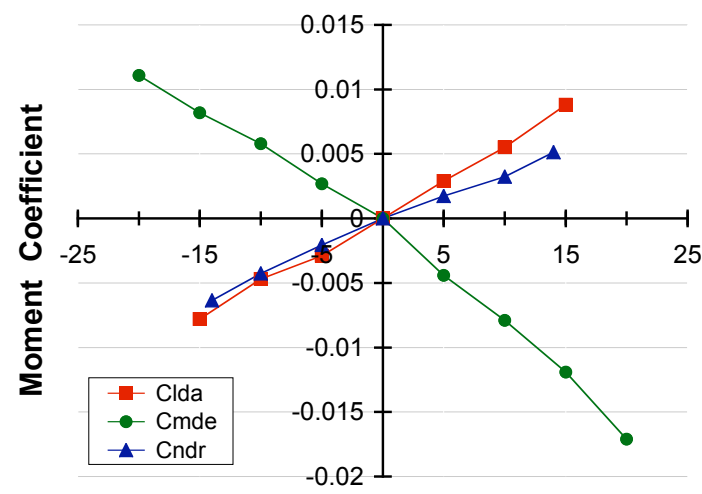

Control Deflection (deg)

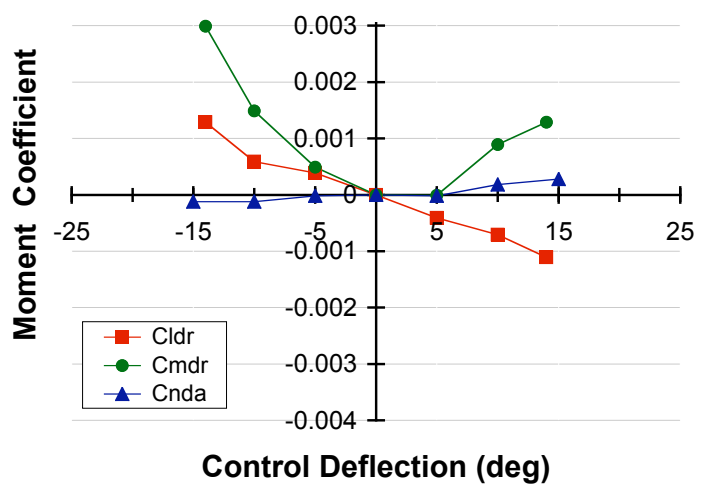

Figure 9. Control moment vs. surface deflection for conventional elevon, aileron and rudder effectors, $\mathrm{AOA}=4 \mathrm{deg}$. 
the 24 shape-change effectors. These are presented in Figure 11, where the effector segments are numbered in ascending order from inboard to outboard, with negative numbers corresponding to the left wing array and positive corresponding to the right wing. Hence, effector segment number -12 designates the outboard-most left wing effector, and +12 designates the outboard-most right wing effector.

Deflection of a single effector device clearly produces useful control moments, but the result is small enough that groups of effectors will be required to generate sufficient moments for typical flight maneuvers. The individual devices were particularly ineffective at generating yawing moment, as indicated by Figures 10 and 11. The low yaw authority of the individual effector segments raises the question of deploying the array in collective fashion intended to generate drag on one wing, and thus yawing moment. Such a configuration was among the six predefined deployment shapes that were evaluated during the next portion of the wind tunnel test.

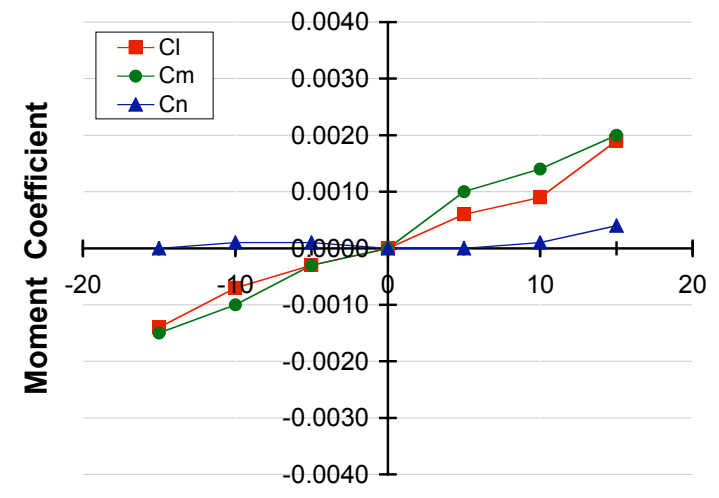

Control Deflection (deg)

Figure 10. Control moment vs. surface deflection for shape-change effector number 6 on the right wing, $\mathrm{AOA}=4 \mathrm{deg}$.

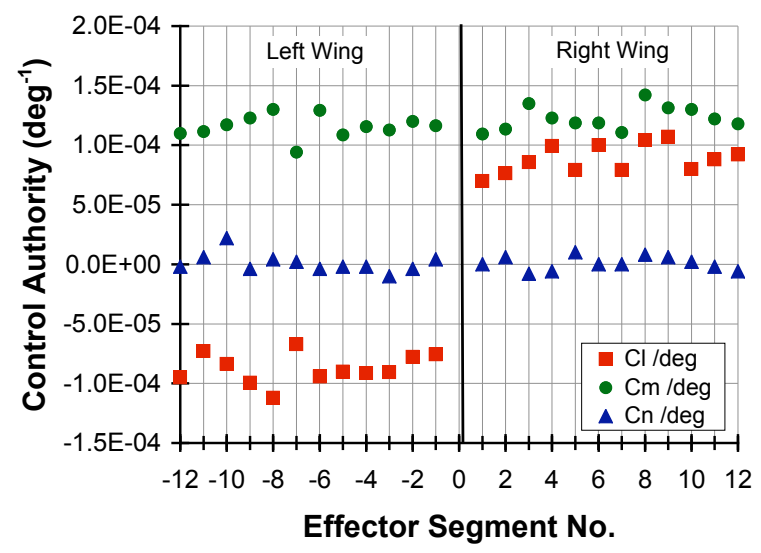

Figure 11. Linearized control authorities for each shapechange device in the trailing edge effector arrays

\section{Control Authorities - Trailing edge Array Shapes}

Control moments were measured for the effector array devices deployed collectively to produce trailing edge shapes, as shown in Figure 5. The shapes could be applied to each wing individually or together in a symmetric or anti-symmetric fashion, and with varying scale factor. A scale factor of 15 corresponded to full deflection of the shape, a condition in which one or more of the individual devices had reached saturation. Figure 12 shows a plot of effector deflection vs. segment number for the six trailing edge shapes applied to the right wing with a scale factor of +15 . The shapes were tested using scale factors ranging from -15 to +15 in increments of 5 .

Figure 13 shows a plot of control moment vs. deflection scale factor for the six trailing edge shapes applied to the right wing only. Not surprisingly, shape 1, which simply sets all devices to a constant deflection, produces the largest pitch and roll moments. But if an objective is to mimic a continuous mold-line effector, then shape 3 appears to generate relatively large moments while satisfying this requirement. Shapes 4 and 6 are capable of generating yaw moment while generating only minimal pitch and roll.

Figures 11 and 13 represent differing approaches to characterizing the authority of the effector array, and their comparison raises important issues regarding methods of control allocation and mixing for effector arrays composed of large numbers of relatively lowauthority devices. One issue is whether allocation should be based on individual device authorities, or rather upon authorities of basis functions representing collective deployment configurations. Both approaches have pros and cons. As devices composing the array become smaller and more numerous, the dimension of the control matrix goes up while individual authority goes down, increasing the potential for ill-conditioned matrices.

Also, in some cases, the devices will only be effective when deployed collectively in a particular combination, and this effect may be missed by an individual device-wise characterization. A good example is the generation of yawing moment. When the yawing moment of the crow mix configuration (shape 6) was predicted by using superposition of the individual linearized device authorities, the result was less than $60 \%$ of the measured authority for that configuration. Presumably, device interactions were important in this configuration, and these effects were not captured by the individual linearized authorities.

A basis function approach appears to provide a means of reducing the high dimensional low authority challenge, but is also likely to lead to difficulty in deriving full authority from the array when a basis function is commanded to saturation. Furthermore, how should such bases be identified? In the MESA experiment the 


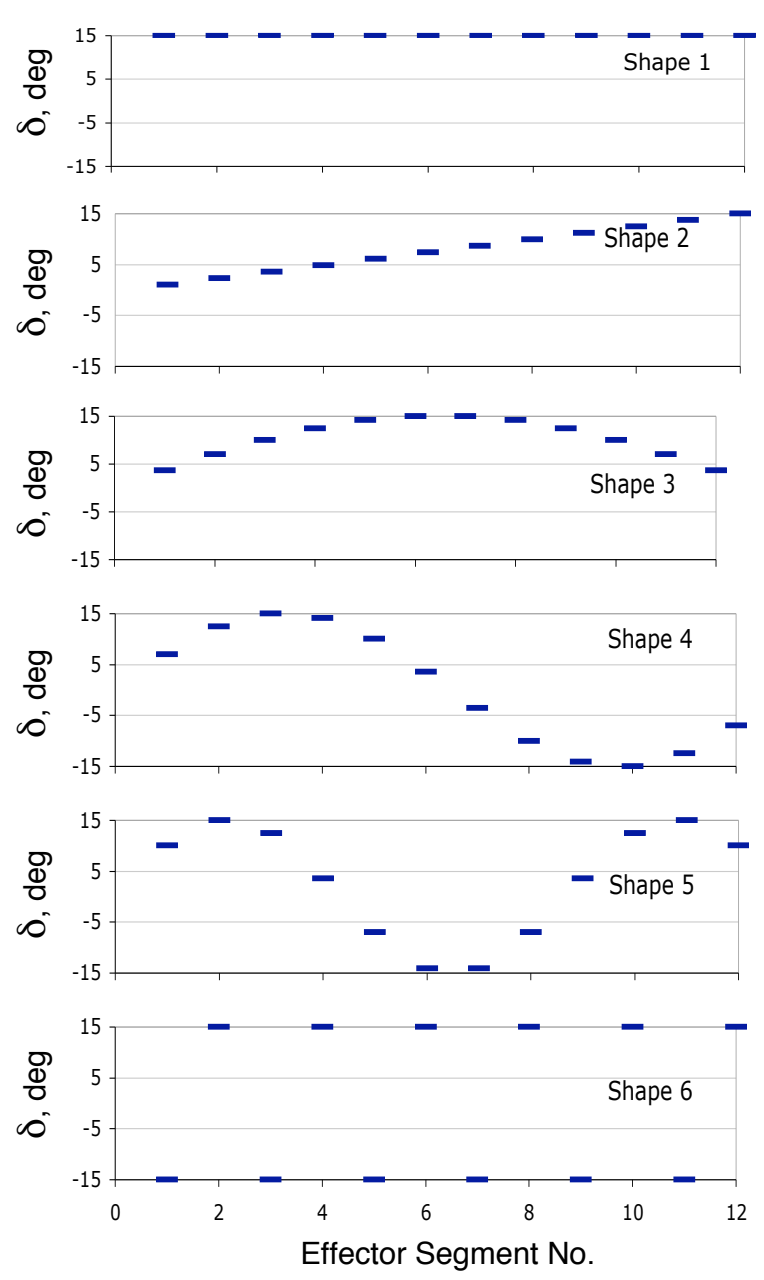

Figure 12. Effector deflection vs. segment number for the six trailing edge shapes applied to the right wing with a scale factor of +15 .

crow mix configuration was selected for shape 6 as a likely yaw generator based upon conjecture. But perhaps alternating in groups of two might have been even more effective.

Hence, there is a need for methodologies to provide thorough and efficient characterization of high dimensional arrays. Whether experimentally or analytically evaluated, the test matrix must explore combinations of device deflections to identify significant nonlinearity and interactions, and the combinatorial possibilities increase rapidly with the number of elements in the array. If the control effector suite of future flight vehicles is truly progressing toward large networks of distributed devices, issues such as allocation, efficient testing, and characterization of high dimensional effector arrays will become increasingly significant. Potential solutions may arise from a blending of information technology concepts with flight control. Decentralized approaches may be envisioned that draw upon principles from collaborative robotics, network theory or cellular automata.
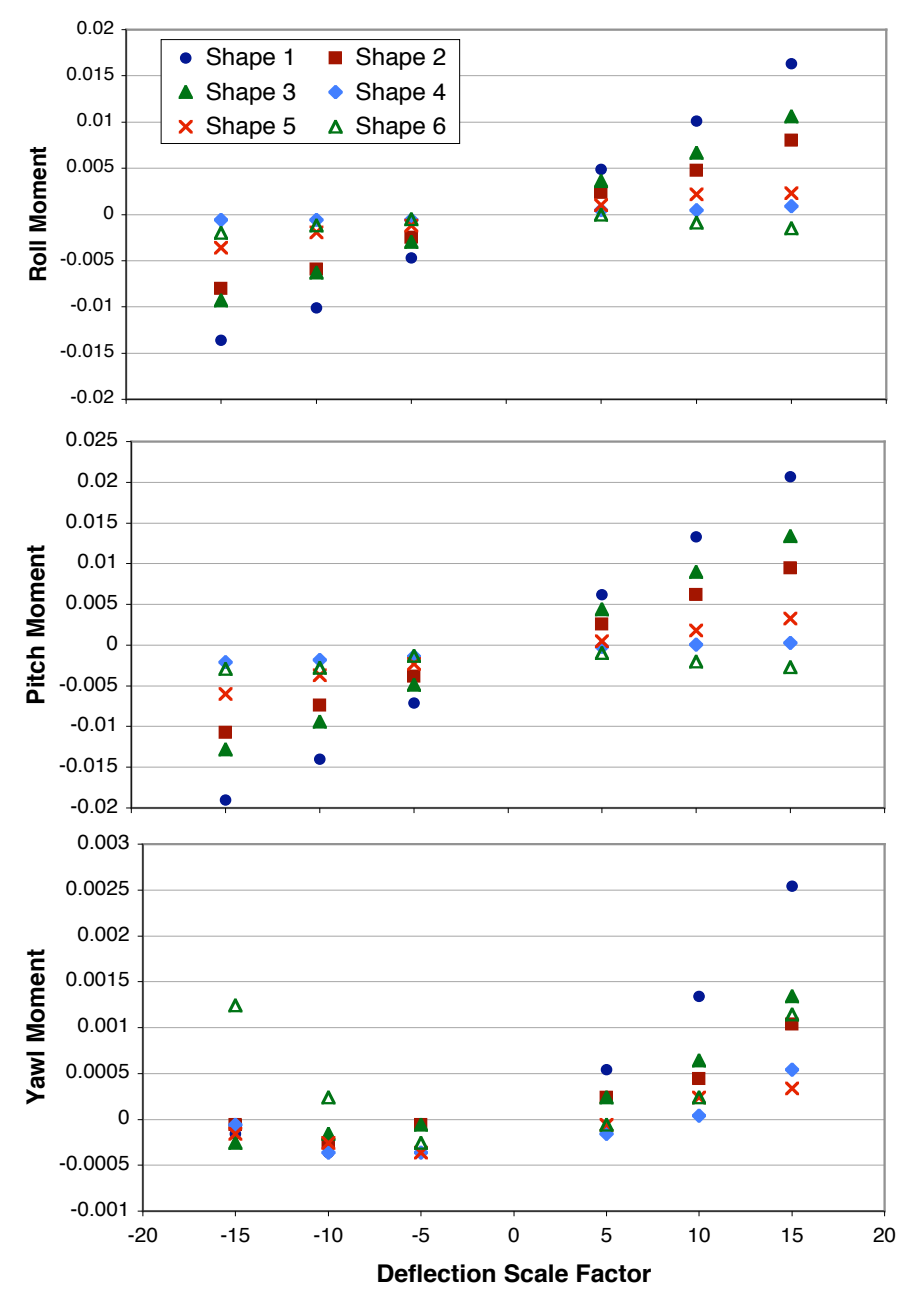

Figure 13. Control moment vs. deflection scale factor for the six trailing edge shapes applied to the right wing, $\mathrm{AOA}=4$ degrees.

\section{PRESSURE MEASUREMENT AND CONTROL}

Variations in the spanwise pressure distribution were measured in response to deflections of the individual trailing edge effectors. The influence of an effector deflection was greatest at the closest pressure measurement, and diminished as distance to the measurement location increased. This trend is clearly visible in the plot of spanwise pressure measurements in response to deflections of +15 and -15 degrees for shape-change effector segment number 4 on the right wing, shown in Figure 14. This plot provides an indication of the degree to which maximum deflection of a single device is able to influence the spanwise pressure distribution.

Pressure measurements such as those shown in Figure 14 were collected for each of the 24 shape-change effectors at settings of $-15,-10,-5,5,10$, and 15 degrees for angles of attack of $0,4,8$ and 12 degrees. The measurements were then used to generate pressure sensitivity matrices for the effector and sensor array using a least- 
squares fit to the pressure variation in response to effector deflection at each sensor location. This process resulted in a separate $12 \times 12$ pressure sensitivity matrix for the right and left wings at each angle of attack.

The pressure sensitivity matrices tended to be diagonally dominant since each device influenced its own pressure measurement most strongly, with diminishing influence on adjacent measurements. A representative sensitivity matrix for the right wing at 4 degrees angle of attack is shown in Figure 15 as a colormap of the sensitivities. The pressure sensitivity matrices were used in the formulation of a feedback control system that was designed to achieve and regulate a commanded spanwise pressure distribution at each of the 24 sensor locations.

\section{Pressure Distribution Control Design}

The MESA testbed was developed to explore the use of multifunctional effector and sensor arrays. A fundamental aspect of the research is to investigate control concepts that exploit the ability of these arrays to simultaneously perform a variety of tasks such as flight

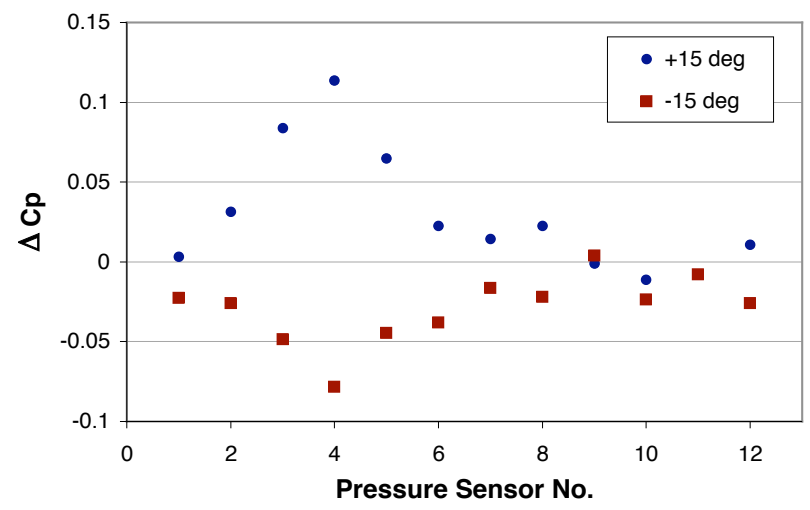

Figure 14. Pressure variations in response to deflections of effector segment 4 on the right wing, $\mathrm{AOA}=4 \mathrm{deg}$.

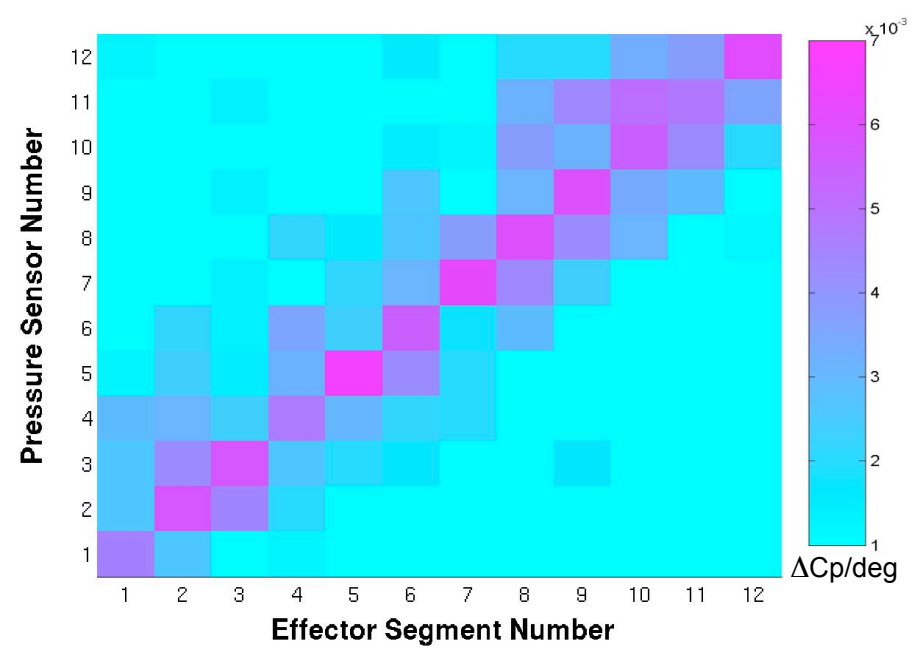

Figure 15. Colormap of pressure sensitivity matrix for right wing effector and sensor array at 4 degrees AOA. control, mission-adaptive performance enhancement, health monitoring, distributed load alleviation, or distributed flutter suppression.

The ability to sense and modify the spanwise pressure distribution acting on the wing would presumably have application to distributed load alleviation and mission-adaptive performance enhancement functions by providing the capacity to tailor the spanwise lift distribution. Such tasks might be assigned a lower priority and performed concurrently with the task of generating moments for flight control purposes. To further examine this possibility a pressure distribution control system that uses the effector and sensor array was developed. The system uses a time domain implementation of the least mean squares (LMS) algorithm ${ }^{12,13}$ to achieve and regulate a commanded spanwise pressure distribution consisting of specified conditions at each of the 24 pressure measurement locations.

For the controller synthesis, let the (n x 1) vector of pressure coefficient variations be given by,

$$
\mathbf{C p}=\mathbf{H d}
$$

where $\mathbf{d}$ is the ( $\mathrm{n} \times 1)$ vector of effector displacements, and $\mathbf{H}$ is the ( $\mathrm{n} \times \mathrm{n}$ ) sensitivity matrix relating effector displacements to pressure variation. The error vector of differences between the measured and desired pressure coefficient vectors is denoted,

$$
\mathbf{e}=\mathbf{C} \mathbf{p}_{\text {desired }}-\mathbf{C} \mathbf{p}_{\text {measured }}
$$

where $\mathbf{C} \mathbf{p}_{\text {measured }}=\mathbf{C p}$ in the case of zero measurement error (no external perturbations, no measurement noise). The nominal control objective is to minimize the error in Eq. 2 subject to constraints on the maximum effector displacement. This is achieved by minimizing the objective function, $J$ :

$$
\begin{array}{ll}
J=E\left\{\mathbf{e}^{T}(n) \mathbf{e}(n)\right\} \quad & \text { subject to } \\
& c_{i}(\mathbf{d})<0, \quad i=1, \ldots n
\end{array}
$$

where $E\{\}$ is the expectation operator, and $c_{i}$ is the $i^{\text {th }}$ convex constraint on the effector displacement vector, $\mathbf{d}$. The control objective function may be rewritten to include the constraints as penalties:

$$
J=E\left\{\mathbf{e}^{T}(n) \mathbf{e}(n)\right\}+\square \square_{i}^{n}\left\{\left[c_{i}(\mathbf{d})\right]_{z}\right\}^{2}
$$

where $\square$ determines the relative importance of the constraints in the objective function. The constraint penalty term, $\left[c_{i}(\mathbf{d})\right]_{z}$ in Eq. 4 is defined by,

$$
\left[c_{i}(\mathbf{d})\right]_{z}=\max \left[c_{i}(\mathbf{d}), 0\right]=\stackrel{\square}{\square} c_{i}(\mathbf{d}) \quad \begin{gathered}
c_{i}(\mathbf{d}) \square 0 \\
\text { otherwise }
\end{gathered}
$$

The LMS algorithm is a stochastic gradient descent procedure for solving a least squares problem, such as 
Eq. 4. At the $k^{\text {th }}$ time step, new values for the displacements are computed according to,

$$
\mathbf{d}(k+1)=\mathbf{d}(k) \square \square \frac{\partial J}{\partial \mathbf{d}}
$$

where $\mu$ determines the rate of adaptation. The LMS algorithm uses an instantaneous estimate of the gradient. This estimate is given by:

$$
\frac{\partial J}{\partial \mathbf{d}}=2 \bigoplus \mathbf{H}^{T} \mathbf{e}+\square \square_{i}^{n}\left[c_{i}(\mathbf{d})\right]_{z} \frac{\partial c_{i}}{\partial \mathbf{d}}=
$$

Let the $i^{\text {th }}$ constraint be defined such that the absolute value of the deflection of the $i^{\text {th }}$ effector must be less than a maximum deflection, $d_{\max }$. Thus,

$$
c_{i}=\left|d_{i}\right|-d_{\max }
$$

Therefore,

$$
\frac{\partial c_{i}}{\partial d_{j}}=\stackrel{\square \operatorname{sign}\left(d_{i}\right)}{ } \begin{array}{ll}
\text { for } \quad i=j \\
0 & \text { otherwise }
\end{array}
$$

With the preceding definitions, the update for the $i^{\text {th }}$ effector displacement is,

$d_{i}(k+1)=d_{i}(k) \square \square\left[\square H(:, i) \llbracket(k)+\square\left[c_{i}\right]_{z} \operatorname{sign}\left(d_{i}\right)\right]$

where $H(:, i)^{\prime}$ ' denotes the transponse of the $i^{\text {th }}$ column of H. After initial simulations of the algorithm a further constraint was added that enforced smoothness of the effector deployment distribution by penalizing large displacements relative to adjacent effectors. The array was thus made to approximate a seamless continuously deformable shape-change trailing edge effector. The update expression was implemented in C-code for realtime control of the model hardware during the wind tunnel test. The shape change deflection angle limit, $d_{\max }$ was set to 15 degrees. The parameters $\square$ and $\square$ were assigned values of 1.0 and 0.1 , respectively.

\section{Pressure Distribution Control Test Results}

The controller was evaluated during the closed-loop portion of the wind tunnel test. A series of pressure distribution commands was created based on known achievable distributions that had been measured during the previous portion of the test. Figure 16 shows an example time history of the commanded and measured pressure coefficients at a single pressure tap as the controller attempted to track a series of step commands. Figure 17 shows a time history colormap of commanded pressure coefficients (top) and measured pressure coefficients (bottom) at all 24 sensor locations from the run shown in Figure 16. Figure 18 shows a 3-d plot of control effector deflections from the same time history.

The LMS controller is clearly effective in commanding the shape changes required to achieve the desired spanwise pressure distribution at the trailing edge sensor array. The relatively long rise time of the closed loop system shown in Figure 16 represents a trade-off between speed of convergence and measurement noise rejection. This trade is controlled by the adaptation rate parameter, $\square$. For this experiment, the parameter was assigned a value of 1.0, resulting in a rise time of approximately 5 seconds, which is sufficient for a flaplike cruise performance optimization function. Future experiments will vary the adaptation parameter to investigate the potential for faster convergence.

The results suggest the potential to employ a similar approach to command larger scale shape changes of a continuously deformable aircraft structure, such as envisioned by the Aircraft Morphing Program, to generate a desired global pressure distribution for mission-adaptive performance optimization or distributed load alleviation purposes. Yet to be addressed is the prioritization and blending of these functions with the multi-axis moment generating functions required for stabilization and maneuver control if the same multifunctional array is used for both purposes.

\section{CONCLUDING REMARKS}

This report has described a project that seeks to investigate controls challenges associated with novel effector and sensor concepts within NASA's Aircraft Morphing Program. In particular, the potential exists to shift the composition of an aircraft's control effector suite from a small number of high authority, specialized devices (rudder, aileron, elevator, flaps), toward arrays composed of larger numbers of smaller, less specialized, distributed effector and sensor devices able to simultaneously perform a variety of control functions using feedback from disparate data sources. To investigate this concept, a remotely piloted flight vehicle has been equipped with an array of 24 trailing edge shape-change effectors and associated pressure measurements to create a representative testbed that embodies the fundamental controls challenges.

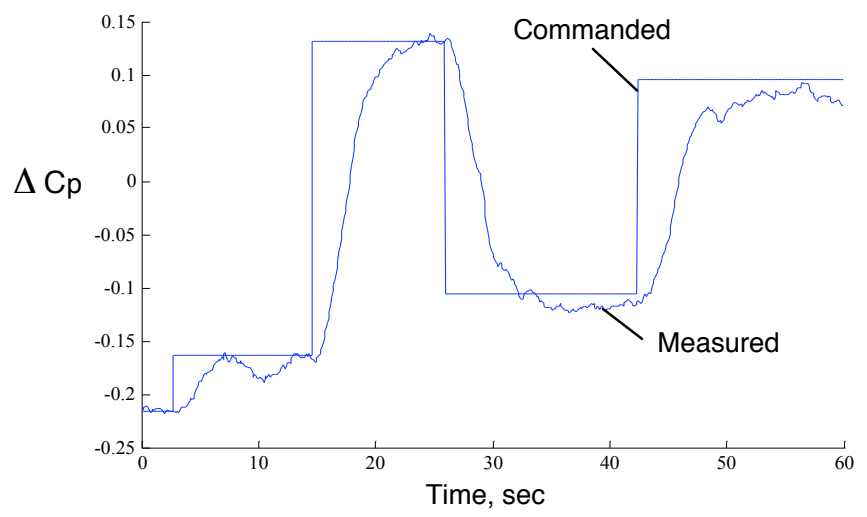

Figure 16. Time history of commanded and measured pressures at a single sensor location, $\mathrm{AOA}=4$ degrees. 


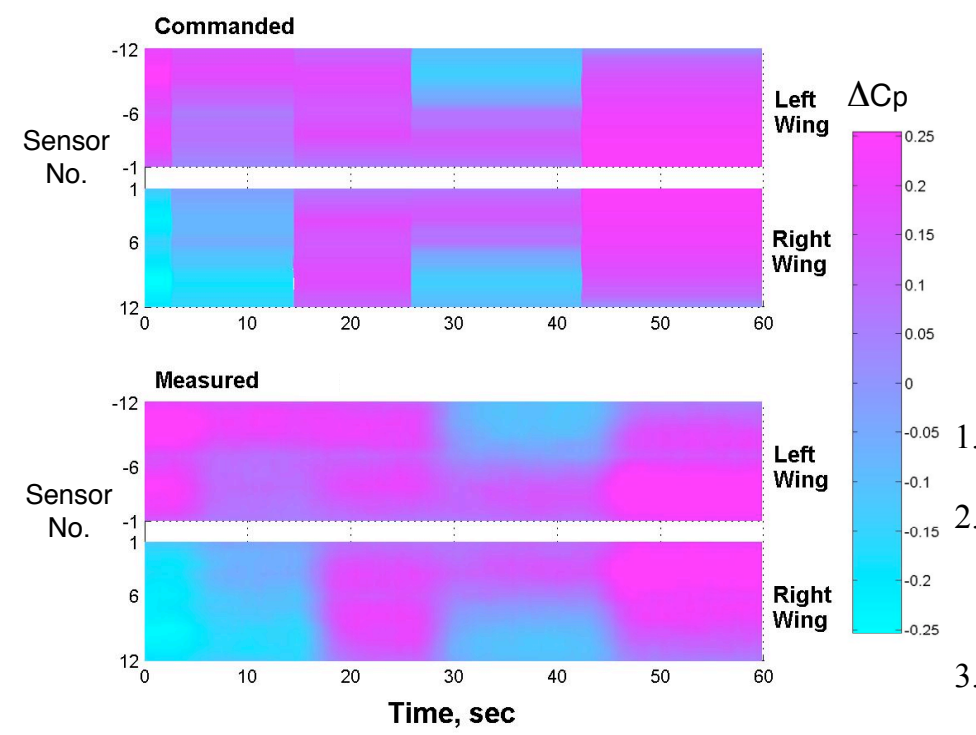

Figure 17. Example time history of commanded and measured pressures at all 24 sensor locations.

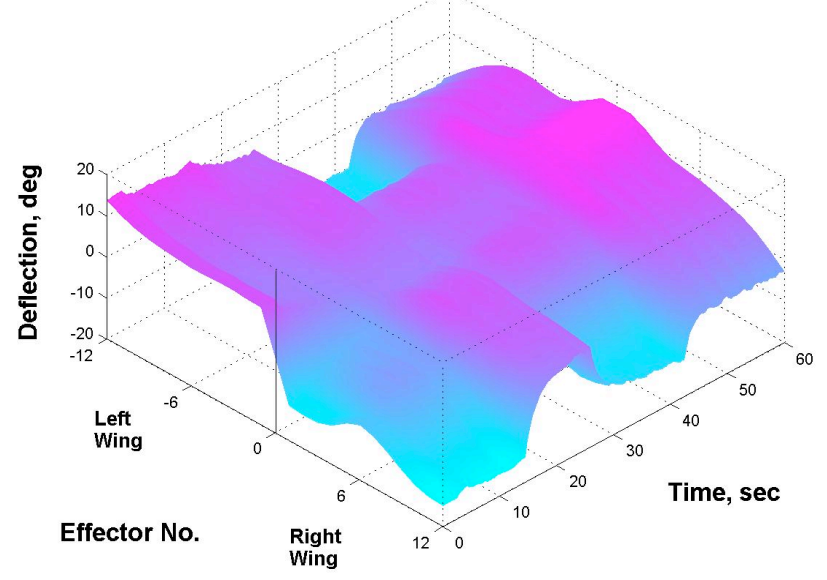

Figure 18. Time history of shape-change effector deflections from pressure distribution controller.

The vehicle, called the Multifunctional Effector and Sensor Array (MESA) testbed, was tested in NASA Langley's 12-ft Low Speed wind tunnel to characterize its stability properties, control authorities, and distributed pressure sensitivities, and to evaluate the design of a spanwise pressure distribution controller that used the model's trailing edge effector and sensor arrays to achieve a commanded spanwise pressure distribution. The design of the pressure distribution controller was described and results from its implementation during the wind tunnel experiment were presented.

The results show that the multifunctional effector and sensor array has the potential to generate sufficient moments for multi-axis flight control purposes as well as to achieve and regulate the spanwise pressure distribution for possible mission adaptive performance optimization or active load alleviation purposes. The control approach offers promise for application to larger scale continuously deformable shape-change configurations such as those envisioned in the Morphing Program. Challenges associated with control characterization and allocation for high dimensional effector arrays were noted and have yet to be addressed. Prioritization and blending of flight control and pressure regulation functions are also topics requiring additional research.

\section{REFERENCES}

Kroo, I.: "Aerodynamic Concepts for Future Aircraft," AIAA Paper 99-3524, July 1999.

2. Park, M.A.; Green, L.L.; Montgomery, R.C.; Raney, D.L.: "Determination of Stability and Control Derivatives Using Computational Fluid Dynamics and Automatic Differentiation", AIAA 99-3136, June 1999.

3. Padula, S.L.; Rogers, J.L.; Raney, D.L.: "Multidisciplinary Techniques and Novel Aircraft Control Systems", AIAA paper 2000-4848, April 2000.

4. Raney, D. L., Montgomery; R. C., Green, L.L.; Park, M. A.: "Flight Control using Distributed Shape-Change Effector Arrays," AIAA paper 2000-1560, April 2000.

5. Bieniawski, S.; Kroo, Ilan M.:"Flutter Suppression Using Micro-Trailing Edge Effectors”, AIAA paper 2003-1941.

6. Lee, Hak-Tae; Kroo, Ilan M.; Bieniawski, S.: "Flutter Suppression for High Aspect Ratio Flexible Wings Using Microflaps", AIAA 2002-1717, 43rd AIAA Structures, Structural Dynamics and Materials Conf, April 2002.

7. Florance, J.P.; Burner, A.W.; G.A.; Hunter, C.A; Graves, S.S.;Martin, C.A.: "Contributions of the NASA Langley Research Center to the DARPA/AFRL/NASA/Northrop Grumman Smart Wing Program”, No. 2003-1961, AIAA Dynamics Specialists Conf, April 2003, Norfolk, VA

8. Martin, C.A., et al.: "Design, Fabrication, and Testing of Scaled Wind Tunnel Model for Smart Wing Phase 2 Program", No. 4332-50, SPIE Symposium on Smart Structures and Materials, Newport Beach, CA, March 2001.

9. Wang, D. P.; Bartley-Cho, J.D.; Martin, C. A.; Hallam, B. J.: "Development of High-Rate, Large Deflection, Hingeless Trailing Edge Control Surface for the Smart Wing Wind Tunnel Model”, Vol. 4332, pp.401-418, SPIE Symposium on Smart Structures and Materials, Newport Beach, CA, 5-8 March 2001.

10. Barnwell, William G.: "Distributed Actuation and Sensing on an Uninhabited Aerial Vehicle", Thesis for Master of Science in Aerospace Engineering at North Carolina State University, MAE Dept, August 2003.

11. Ashby, D. L., Dudley, M. R., Iguchi, S. K., Browne, L., Katz, J., "Potential Flow Theory and Operation Guide for the Panel Code PMARC," NASA TM-102851, Jan 1991.

12. Haykin, S.: Adaptive Filter Theory. Prentice Hall, 1991, Englewood Cliffs, NJ; ISBN 0-13-013236-5.

13. Rafaely, B.; Elliott, S. J.: "A Computationally Efficient Frequency-Domain LMS Algorithm with Constraints on the Adaptive Filter", IEEE Transactions on Signal Processing, Vol. 48, No. 6, June 2000, pp. 1649-1655. 